\title{
Wavelet Package Energy Transmissibility Function and Its Application to Wind Turbine Blade Fault Detection
}

DOI:

10.1109/TIE.2022.3146535

\section{Document Version}

Accepted author manuscript

Link to publication record in Manchester Research Explorer

\section{Citation for published version (APA):}

Wang, X., Liu, Z., Zhang, L., \& Heath, W. (2022). Wavelet Package Energy Transmissibility Function and Its Application to Wind Turbine Blade Fault Detection. IEEE Transactions on Industrial Electronics. https://doi.org/10.1109/TIE.2022.3146535

\section{Published in:}

IEEE Transactions on Industrial Electronics

\section{Citing this paper}

Please note that where the full-text provided on Manchester Research Explorer is the Author Accepted Manuscript or Proof version this may differ from the final Published version. If citing, it is advised that you check and use the publisher's definitive version.

\section{General rights}

Copyright and moral rights for the publications made accessible in the Research Explorer are retained by the authors and/or other copyright owners and it is a condition of accessing publications that users recognise and abide by the legal requirements associated with these rights.

\section{Takedown policy}

If you believe that this document breaches copyright please refer to the University of Manchester's Takedown Procedures [http://man.ac.uk/04Y6Bo] or contact uml.scholarlycommunications@manchester.ac.uk providing relevant details, so we can investigate your claim.

\section{OPEN ACCESS}




\title{
Wavelet Package Energy Transmissibility Function and Its Application to Wind Turbine Blade Fault Detection
}

\author{
Xuefei Wang, Student Member, IEEE, Zepeng Liu, Member, IEEE, \\ Long Zhang, Member, IEEE, and William P. Heath, Member, IEEE
}

\begin{abstract}
To harvest wind energy from nature, wind turbines are increasingly installed globally, and the blades are the most essential components within the turbine system. The blades usually suffer from time-varying non-stationary wind loads, and the load information is normally unknown or difficult to collect. This poses significant challenges to the blade assessment and damage detection. Transmissibility Function (TF) methods have the potential to address this challenge as they do not require loading information. In this paper, a novel Wavelet Package Energy TF (WPETF) method is proposed to increase the high frequency resolution while maintaining its low sensitivity to noise and it is further used for wind turbine blade fault detection. Compared with the existing Fourier TF (FTF) method, the proposed method is immune to the external loading impacts, does not require excitation knowledge, and is robust to noise. Compared with the existing Wavelet Energy TF (WETF) method, the novel one uses Wavelet Package Decomposition (WPD) instead of Wavelet Decomposition (WD) to further increase the high frequency resolution which provides richer damage-induced information. The effectiveness of the WPETF method for wind turbine blade condition assessment is first verified numerically and then on three industrial-scale wind turbine blades with both naturally (uncontrolled) and artificially-introduced (controlled) damage. Its advantages over a number of existing methods are also demonstrated.
\end{abstract}

Index Terms-Wind turbine blade, Condition monitoring, Fault detection, Vibration analysis, Wavelet transmissibility function

\section{INTRODUCTION}

$\mathbf{I}^{\mathbf{N}}$ $\mathrm{N}$ wind power systems, wind turbines are essential to transform kinetic energy to electricity, and the blades play a crucial role in capturing natural wind energy and are costly components [1]. Especially, for a modern 10 megawatt wind turbine, the length of its blade has reached $88.4 \mathrm{~m}$ which is longer than the wingspan of any current aircraft including Boeing 787 [2]. However, the blades usually work under time-varying wind loads and may also suffer from extreme weather conditions such as wind gusts, storms and lightning, in particular for those turbines in harsh remote areas including offshore sites [3]. This may shorten their fatigue life and even lead to premature damage. Typical wind turbine blade

Xuefei Wang, Long Zhang and William P. Heath are with the Department of Electrical and Electronic Engineering, The University of Manchester, Manchester, M1 3BB, U.K. (e-mail: xuefei.wang@manchester.ac.uk, long.zhang@manchester.ac.uk, william.heath@manchester.ac.uk)

Zepeng Liu is with the Department of Automatic Control and Systems Engineering, University of Sheffield, Sheffield, S10 2TN, U.K. (e-mail: zepeng.liu@ sheffield.ac.uk) Corresponding author: Long Zhang faults occur during both production and operation stages. Production defects appear during the design and manufacture stage, including cracks, delamination, debonding, wrinkle and buckling. Operational failures are identified mainly during the in-service period, and these failures are usually caused by external environmental factors. Operational defects include ice or sand accumulation, erosion, corrosion, lightning damage, dust and insect contamination [4]. It is noteworthy that some production defects could also occur during the operational stage, such as cracks and delamination. If the incipient damage is not found at an early stage, it could propagate and further result in severe malfunction, fatal damage, or the worst case of the whole turbine collapse, which usually causes a significant financial loss [4]. Statistics show that the blades are easily exposed to damage and have a high failure rate [5]. The development of corresponding wind turbine blades damage detection techniques is hence essential to prevent severe damage, associated economic loss, and further reduce the operation and maintenance cost.

Various wind turbine blade damage detection techniques have been developed over the past two decades. The primary state-of-the-art methods are the non-destructive detection solutions, such as acoustic emission [6], strain [7] or bending moment measurement [8], infrared thermography [9], ultrasonic detection [10], and vibration analysis [11]. However, the majority of these attempts still remain at the laboratory stage and have not been widely tested and installed into in-situ applications yet. Vibration-based methods are extensively studied to detect the blade faults, with natural wind force or external force like hammer tapping [12]. These dynamic vibration responses are often used as features for blade condition assessment [11]. Machine/deep learning methods are applied to vibration-based online rotational blade fault detection [13], [14]. Signal processing techniques, such as frequency domain analysis, time domain analysis, and frequency-time analysis, are used to abstract blade damage signatures from vibrational responses [15], [16]. Support vector machines (SVM), hidden Markov Models (HMM), finite element method (FEM) are also used as the vibrational signal processing tools to identify blade damage [17], [18]. A more comprehensive review of vibration-based blade fault detection techniques can be found in [19], and this paper mainly focuses on vibration-based detection methods.

It is essential to address the following challenges when detecting wind turbine blade faults. Firstly, in-service blades 
are subjected to the non-stationary time-varying wind loads [19]. Normally, the external loading information is unknown or sometimes difficult to measure [20]. Secondly, both environmental and machine noise may dramatically reduce the blade faults detection accuracy [3]. Finally, the detection technique has to be sensitive enough to various modes of defects and applicable to different types of blades while remaining insensitive to noise [21].

As a promising method, Frequency Response Function (FRF) is able to eliminate the loading impact which is defined as the spectra ratio between the measurement responses and excitation pairs. The excitation or input loading effects are therefore removed by the ratio operation [22]. However, the input loading information of the blades is often not available, which makes the FRF hard to be applied for condition assessment purposes. Similar to FRF, Transmissibility Function (TF) is defined as the ratio of two individual output responses, which only requires system responses without the usage of loading or excitation information [4]. Conventionally, TF is estimated using Fourier transform, however, the Fourier TF (FTF) method is sensitive to noise and is not fully immune to loading variations [23]. To deal with these two issues, a Wavelet Energy TF (WETF) method has been proposed, which carries out wavelet decomposition (WD) of two individual measured responses and then calculates the ratio of the wavelet energy. WETF shares the same time-frequency resolutions with WD [24]. For WD, it has higher resolution in low frequency zones but has lower resolutions in high frequency zones. For some applications such as blade fault detection, their fault condition features may exist in a certain high frequency range. The WETF may have limitations in detecting those defects effectively. To deal with this issue, the main contributions of this paper are summarized as follows:

- A novel algorithm, the Wavelet Package Energy TF (WPETF) method, is proposed as a new time-frequency function. The new function is estimated using output only information and it does not require loading or excitation information. Compared to the original WETF method, the new method has a higher frequency resolution. When it is used for fault detection, it will provide richer information of fault and make it easier to distinguish healthy and damaged conditions.

- The new method can be used for a wide range of engineering systems or structure fault detection. The superiority of the new method over the existing WETF in terms of sensitivity to system property change is first demonstrated using a numerical mass-damping-spring system. The higher sensitivity could avoid missing early fault detection or provide better fault detection accuracy.

- To demonstrate the practical use of the new method, it is applied to three industrial-scale wind turbine blade fault detection. Extensive results from both naturally damaged (cracks and delamination) and controlled damaged (local and large area ice/sand accretions) cases demonstrate the reliability and effectiveness of the new method in terms of both accuracy and robustness.

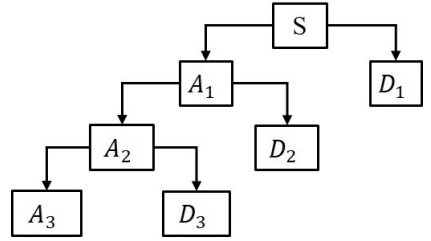

Fig. 1 3-layer wavelet decomposition tree structure

The rest of this paper is organized as follows: Section II introduces the WETF methods, and section III proposes the new WPETF method. Section IV demonstrates the superiority of WPETF over WETF via numerical example simulations. Section V shows the experiments setup, procedures, results analysis and validations. Section VI lists some related discussions. Section VII concludes the paper.

\section{WAVELET ENERGY TRANSMISSIBILITY FUNCTIONS}

The wavelet transform decomposes the measured signal into a series of wavelet functions acting as low pass and high pass filters [25]. For a Multiple Degrees of Freedom (MDOF) linear system, for example, a wind turbine blade structure, its dynamic representation can be delivered as [26]

$$
\mathbf{M} \ddot{s}+\mathbf{P} \dot{s}+\mathbf{Q} s=\mathbf{S}
$$

where $s$ denotes the displacement responses along the blade. $\mathbf{M}, \mathbf{P}$, and $\mathbf{Q}$ represent the mass, damping and stiffness matrices of the blade structure respectively. Suppose the system has $n$ responses, denoted as $\mathbf{S}=\left[S_{1}, \ldots, S_{i}, \ldots, S_{n}\right]^{T}$ with

$$
S_{i}=\left[s_{1}^{i}, \ldots, s_{r}^{i}, \ldots s_{N}^{i}\right]
$$

Each $s_{i}$ stands for the single system output from the $i$ th output response. $N$ is the data length within each response.

For 1-layer wavelet decomposition, the original signal $S_{i}$ can be decomposed into a detailed part $D_{1}^{i}$ with a high pass filter $g$, and an approximation part $A_{1}^{i}$ with a low pass filter $h$. This process can be denoted as

$$
\begin{aligned}
& D_{1}^{i}=\left\{\sum_{k} s_{k}^{i} g_{q-k}, q=1, \ldots, N\right\} \downarrow 2 \\
& A_{1}^{i}=\left\{\sum_{k} s_{k}^{i} h_{q-k}, q=1, \ldots, N\right\} \downarrow 2
\end{aligned}
$$

where $\downarrow 2$ indicates that the filtered results are downsampled by a factor of 2. For 2-layer decomposition, the approximation $A_{1}^{i}$ is further decomposed with the same procedure. The procedure can continue for $J$-layer decomposition. As an example, Fig.1 displays the tree structure of a 3-layer WD, where $S$ stands for the initial raw signal. For a $J$-level decomposition, the coefficients are given as

$$
\begin{aligned}
W^{i} & =\left\{A_{J}^{i}, D_{J}^{i}, D_{J-1}^{i}, \ldots, D_{1}^{i}\right\} \\
& =\left\{\nu_{r}^{i}, r=1, \ldots, R\right\}
\end{aligned}
$$

The WETF is defined as the ratio of wavelet energy between the WD of two independent measured responses. The wavelet energy is defined as the Root Mean Squares (RMS) of a series of WD coefficients.

The total $R$ wavelet coefficients in equation (5) are first divided into $Z$ segments and each segment has $m$ coefficients, 
therefore, $R=Z m$. The wavelet energy $e_{z}^{i}$ for the $z$ th segment is the RMS value of wavelet coefficients from the $z$ th segment data, where $z=1, \ldots, Z$.

$$
\begin{aligned}
e_{z}^{i} & =R M S\left(\nu_{(z-1) m+1}^{i}, \ldots, \nu_{z m}^{i}\right) \\
& =\sqrt{\frac{1}{m} \sum_{h=(z-1) m+1}^{z m}\left(\nu_{h}^{i}\right)^{2}}
\end{aligned}
$$

The WETF, defined as the ratio of wavelet energy from two different measured responses, say $i$ th and $j$ th responses $(i, j=$ $1, \ldots, n, i \neq j$ ), is given by

$$
T^{i j}=\left[\frac{e_{1}^{i}}{e_{1}^{j}}, \ldots, \frac{e_{z}^{i}}{e_{z}^{j}}, \ldots, \frac{e_{Z}^{i}}{e_{Z}^{j}}\right]=\left[\mu_{1}^{p}, \ldots, \mu_{z}^{p}, \ldots, \mu_{Z}^{p}\right]
$$

where $p=1, \ldots, P$, and the total number of WETF is $P=$ $(n-1) n / 2$. The values of all the transmissibility functions can be rewritten as a matrix form:

$$
T=\left[\begin{array}{cccc}
\mu_{1}^{1} & \mu_{2}^{1} & \cdots & \mu_{Z}^{1} \\
\mu_{1}^{2} & \mu_{2}^{2} & \cdots & \mu_{Z}^{2} \\
\vdots & \vdots & \vdots & \vdots \\
\mu_{1}^{P} & \mu_{2}^{P} & \cdots & \mu_{Z}^{P}
\end{array}\right]
$$

For damage detection, it is necessary to compare the operating TFs with the reference TFs (i.e. from the healthy conditions). The transmissibility correlation $T C_{z}$ is used to quantify the damage severities between healthy condition $\mu$ and operational condition $\bar{\mu}$ at data segment $z$, which is given by

$$
T C_{z}=\frac{\left|\sum_{p=1}^{P} \mu_{z}^{p} \bar{\mu}_{z}^{p}\right|^{2}}{\sum_{p=1}^{P} \mu_{z}^{p} \mu_{z}^{p} \sum_{p=1}^{P} \bar{\mu}_{z}^{p} \bar{\mu}_{z}^{p}}
$$

Algorithm 1 briefly summarizes the procedure of the WETF method.

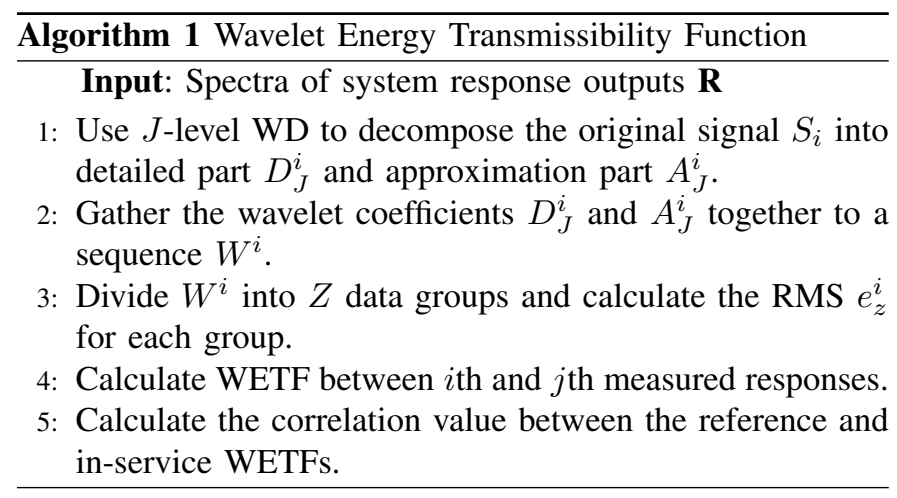

\section{WaVelet Package Energy TRANSMISSIBILITY FUNCTION METHOD}

For the WETF method, its resolution in high frequencies is lower than those in low frequencies. This is essentially the same with the results of the original wavelet transform as the WETF method uses WD as its time-frequency analysis. The popular fast WD method uses low pass and high pass

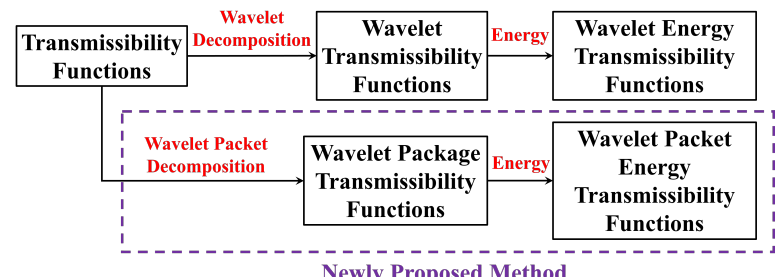

Newly Proposed Method

Fig. 2 Relation between newly proposed method and the existing transmissibility methods

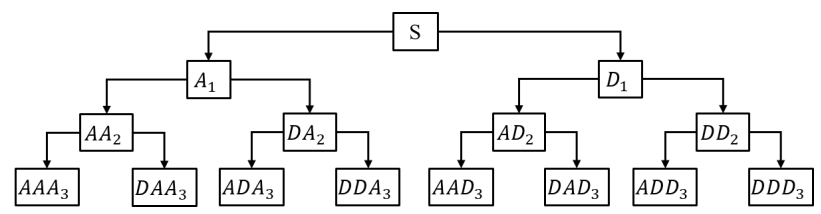

Fig. 3 3-layer wavelet packet decomposition tree structure

filters to separate the signal and carry out fewer levels of decomposition for high frequency signals and therefore it gives lower resolutions for high frequency parts. This could meet the needs of most engineering applications where the noisy information lies in the high frequencies and does not need the same resolution as the low frequencies. However, if the applications, such as the wind turbine blade fault diagnosis with different types of faults, may have useful information in the high frequency parts, the high frequency resolution has to be improved. The Wavelet Packet Decomposition (WPD) can improve the high frequency resolution as it produces the same number of decompositions for both low and high frequencies. In this paper, an extended method, the Wavelet Packet Energy TF (WPETF) method, is proposed to increase the resolution of high frequency ranges of the WETF method. Fig.2 illustrates the relation between the WPETF and the WETF method.

The mechanism for WPD is to decompose both approximated and detailed parts in each layer of decomposition, instead of solely approximated part for WD. Mathematical definition and associated theories of WPD can be found in [27]. Fig.3 illustrates a 3-layer WPD progress. Therefore, for a $J$-layer decomposition, WD only generates $J+1$ frequency zones, but WPD generates $2^{J}$ frequency zones. For high frequency zones, the detailed WPD parts are given by

$$
D_{J}^{i}=\left\{\sum_{k} s_{k}^{i} \gamma_{q-k}, q=1, \ldots, N\right\} \downarrow 2
$$

where $\gamma$ represents a WPD filter synthesized by high pass filter $g$ and low pass filter $h$ [27].

To further illustrate the difference, Fig.4(a) indicates that for the same decomposition layers, the resolution in the low frequency range remains the same, but WPD has higher resolution than WD in the high frequency range. If both WETF and WPETF produce the same number of frequency zones, as shown in Fig.4(b) with the case of 4 frequency zones. It is worth highlighting that the WPETF needs to have 2-layer decomposition and produce evenly distributed frequency zones, while the WETF can have 3-layer decomposition but 


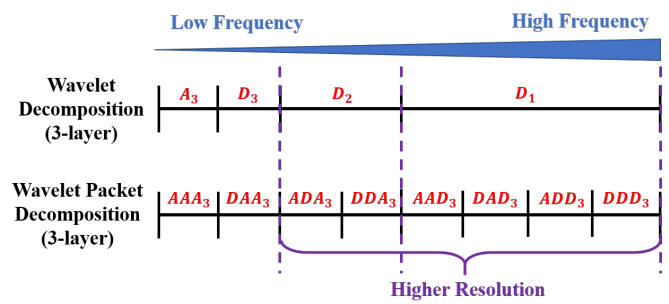

(a) Same number of decomposition layers

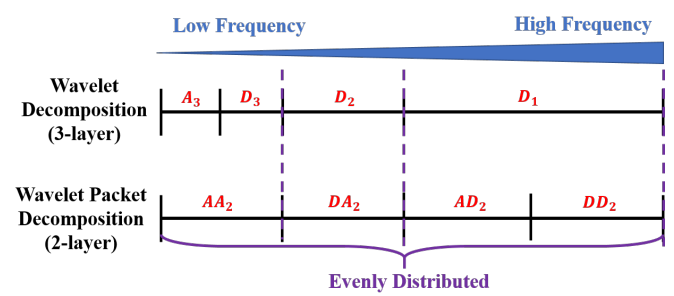

(b) Same number of frequency zones

Fig. 4 Resolution comparison between wavelet and wavelet packet decomposition with (a) same layers and (b) same frequency zone numbers

has unevenly distributed zones with more zones in the low frequencies.

A $J$-layer WPD coefficients are gathered in a sequence, similar as equation (5):

$$
\begin{aligned}
W P^{i} & =\left\{W P_{1}^{i}, W P_{2}^{i}, \ldots, W P_{2^{J}}^{i}\right\} \\
& =\left\{\beta_{r}^{i}, r=1, \ldots, R\right\}
\end{aligned}
$$

where $R$ represents the number of WPD coefficients. These $R$ coefficients are divided into $Z$ groups with $m$ coefficients per group, namely, $R=Z m$. The RMS value for each group as the wavelet energy is calculated by where the RMS is detailed as

$$
\begin{aligned}
d_{z}^{i} & =\operatorname{RMS}\left(\beta_{(z-1) m+1}^{i}, \ldots, \beta_{z m}^{i}\right) \\
& =\sqrt{\frac{1}{m} \sum_{r=(z-1) m+1}^{z m}\left(\beta_{r}^{i}\right)^{2}}
\end{aligned}
$$

The WPETF, defined as the ratio of wavelet package energy from two different measured responses, say $i$ th and $j$ th responses $(i, j=1, \ldots, n, i \neq j)$, is given by

$$
T^{\prime i j}=\left[\frac{d_{1}^{i}}{d_{1}^{j}}, \ldots, \frac{d_{z}^{i}}{d_{z}^{j}}, \ldots, \frac{d_{Z}^{i}}{d_{Z}^{j}}\right]=\left[\xi_{1}^{p}, \ldots, \xi_{z}^{p}, \ldots, \xi_{Z}^{p}\right]
$$

where $p=1, \ldots, P$ and $P=(n-1) n / 2$ as the total number of WPETFs. Similarly, the two-dimension WPETFs can be expressed in a matrix form as:

$$
T^{\prime}=\left[\begin{array}{cccc}
\xi_{1}^{1} & \xi_{2}^{1} & \cdots & \xi_{Z}^{1} \\
\xi_{1}^{2} & \xi_{2}^{2} & \cdots & \xi_{Z}^{2} \\
\vdots & \vdots & \vdots & \vdots \\
\xi_{1}^{P} & \xi_{2}^{P} & \cdots & \xi_{Z}^{P}
\end{array}\right]
$$

The WPETF method is proposed to add higher resolution to the frequency zones of interest which may provide richer information related to blade damage. Algorithm 2 briefly summarizes the procedure of the WPETF method.

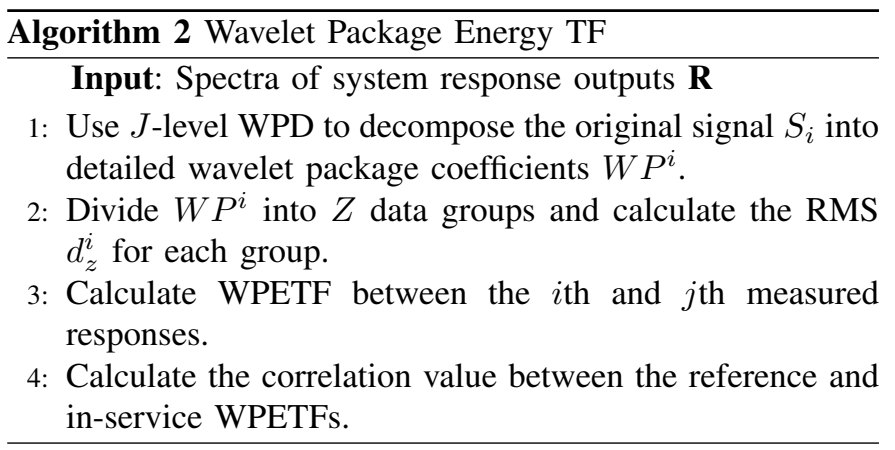

The main advantage of the new WPETF method lies in the increased resolutions for the high frequency parts. Meanwhile, the WPETF inherits the advantage of WETF regarding to the low sensitivity to noise. An analysis for this is provided in Appendix. In addition, the computational complexity comparison between WETF and WPETF is provided here. As the major computations of these two methods are related to WD and WPD, the computational complexity of WD and WPD are directly compared. Assume the computations consumed for a single layer wavelet decomposition is $\Delta$ $(\Delta>0)$. Note that for one layer decomposition, WPD and WD consume the same computations.

- Same decomposition layers: For a $J$-layer decomposition $(J \geq 2)$, WPD carries out the decomposition for both detailed and approximated parts in each layer, and it consumes $W P D_{c s}=J \Delta$. However, WD only carries out the decomposition for the detailed parts in each layer and its computational consumption is

$$
W D_{c s}=\sum_{j=1}^{J} \frac{\Delta}{2^{j-1}}
$$

The difference between them is:

$$
W P D_{c s}-W D_{c s}=\Delta\left[(J-2)+\frac{1}{2^{J-1}}\right]>0
$$

Equation (16) shows that the increased computations are proportional to the number of the decomposition layers. For example, WPD requires $1.25 \Delta$ more computations for a 3-layer decomposition while it consumes $2.125 \Delta$ more computations for a 4-layer decomposition. The increased computations for WPD are mainly used to increase the high frequency resolutions,since WPD has $2^{J}$ frequency zones and WD only has $J+1$ zones for the same $J$-layer decomposition.

- Same number of frequency zones: For the $2^{J}(J \geq$ 2) frequency zones, WPD has $J$ layers decomposition leading to $W P D_{c s}^{\prime}=J \Delta$ computations. However, WD needs $2^{J}-1$ layers decomposition, and the corresponding computations are

$$
W D_{c s}^{\prime}=\sum_{j=1}^{2^{J}-1} \frac{\Delta}{2^{j-1}}
$$

The difference between two decomposition is:

$$
W P D^{\prime}{ }_{c s}-W D^{\prime}{ }_{c s}=\Delta\left[(J-2)+\frac{1}{2^{2^{J}-1}}\right]>0
$$


TABLE I Comparisons between FTF, WETF and WPETF methods

\begin{tabular}{c|ccc}
\hline Criteria & FTF & WETF & WPETF \\
\hline High frequency resolution & highest & low & higher \\
Noise rejection & poor & good & good \\
Computational complexity & most complex & complex & more complex
\end{tabular}

Since $2^{J}-1>J$, for the same number of frequency zones, WD requires more decomposition layers. For example, for a 3-layer WPD, WD needs 7 layers decomposition to reach the same 8 frequency zones, and WPD consumes approximately $\Delta$ more resources. For a 4-layer WPD, WD needs 15 layers to generate 16 frequency zones, and WPD increases the computational burden by approximately $2 \Delta$.

To understand the new WPETF method better, Table I presents the comparisons between FTF, WETF and WPETF in terms of three criteria including high frequency noise rejection, computational complexity and high frequency resolution. The new WPETF enjoys the high resolutions of high frequencies and remains low sensitivity to noise, but uses some additional computations.

\section{Simulation Analysis}

To demonstrate the effectiveness of WPETF method, a mass-damping-spring system is introduced as a numerical example to illustrate the superiority over conventional $\mathrm{TF}$ methods. Suppose one TF in Laplace domain describing the relation between two individual responses [28]:

$$
T F(s)=\frac{36 s+400}{s^{2}+36 s+400}
$$

The first measurement is set by a combination of a series of sine waves over the frequency range from $0 \mathrm{~Hz}$ to $50 \mathrm{~Hz}$ with a resolution of $0.2 \mathrm{~Hz}$. The sampling frequency and data sampling number are set to be $1.2 \mathrm{k} / \mathrm{s}$ and 600 , respectively. The second measurement is the response of the TF function given the input of the first measurement. The WETF and WPETF methods are used to estimate the given TF using the two measurements. To produce results of WETF and WPETF, the filters used in both methods have to be chosen. The low-pass $h$ and high-pass decomposition filter $g$ is set to be $[0,0,0,0,0.1768,0.5303,0.5303,0.1768,0,0,0,0]$ and $[0.0138,0.0414,-0.0525,-0.2679,0.0718,0.9667,-0.9667$, $-0.0718,0.2679,0.0525,-0.0414,-0.0138]$, respectively. The $\gamma$ filter for WPD is set to be $[0.0362,0.0461,-0.0325$, $-0.0749,0.0422,0.0676,-0.0105,-0.0356,0.0747,0.0661$, $-0.0537,-0.0889]$. To apply the energy concept, the resultant wavelet coefficients are grouped. Here, the number of the wavelet coefficients in each group, $m$, is chosen as 1000. The RMS values of each group and the ratio of RMS values between two different measurements are then calculated within 5-layer decomposition of WD and WPD. Fig.5(a) and Fig.5(b) demonstrates the results of WETF and WPETF methods of the given numerical example. Six different levels are classified in terms of the WETF method. However, within high frequency

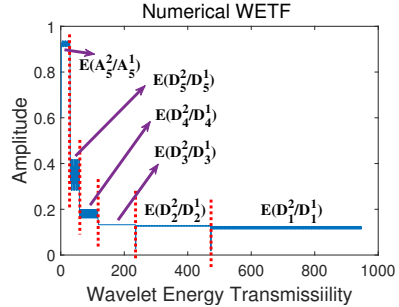

(a)

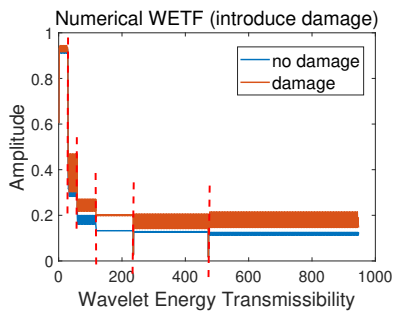

(c)

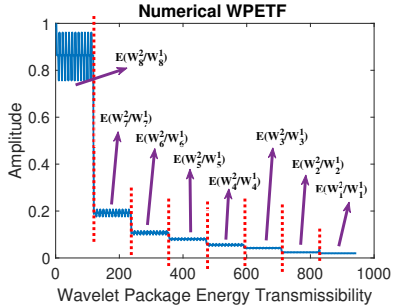

(b)

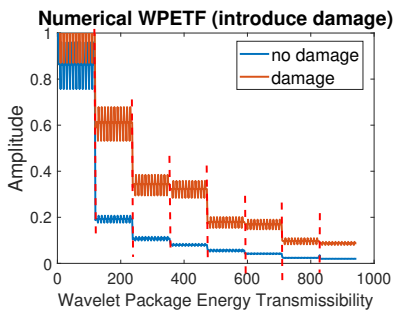

(d)
Fig. 5 Numerical example: WETF and WPETF methods simulation results

zones, levels vary slightly from each other. WPETF method is then proposed to divide the high frequency zone into more detailed sub-frequency zones with more resolution levels, which offers richer details and information. It can be seen that the WPETF result contains more details of the high frequency range and adds more resolutions which could provide more information than the WETF method. To further demonstrate the advantage of the WPETF technique, a second-order is added to the original numerical example to simulate a damaged scenario:

$$
T F_{\text {damage }}(s)=\underbrace{\frac{36 s+400}{s^{2}+36 s+400}}_{\text {system }} \times \underbrace{\frac{90000}{s^{2}+60 s+90000}}_{\text {damage }}
$$

Fig.5(c) and Fig.5(d) illustrates the comparison results of both normal and damaged cases. Clearly, due to the fact that WPETF provides higher resolution in high frequency range, the WPETF method is able to produce more significant amplitude changes due to the damage in the high frequency range than the WETF method.

\section{EXPERIMENTS AND RESULTS ANALYSIS}

The following experiments are designed and conducted to demonstrate the effectiveness of the proposed WPETF method for wind turbine blade condition assessment. The experiments use three naturally damaged industrial-scale blades (per length: 7.75m; per weight: $139 \mathrm{~kg}$ ) from the Wind Turbine Laboratory at the University of Manchester, where blade \#1 is in relatively healthy condition and blade \#2 \& \#3 are damaged. The three blades (type: MOC15150) were manufactured by NOI Scotland Ltd. company in 2002. Fig.6(a)-6(b) show the configuration of the integrated test-rig. The hammer used in the experiments is RS PRO Medium Carbon Steel Lump Hammer $(1.8 \mathrm{~kg})$, and the accelerometer type vibration sensor is Hansford HS-100-type sensor with the following parameters: the frequency response of $2 \mathrm{~Hz}-10$ 
$\mathrm{kHz}$, the sensitivity of $1000 \mathrm{mV} / \mathrm{g}$ and the bias voltage of 10-12 $V_{D C}$. The vibration amplifier HS-511 is responsible for sensor powering, bias voltage and measured responses stripping off. Also, the HS-511 is wired to a high-speed Data Acquisition (DA) device. In this work, we use DAQami software and hardware system to collect the vibration signals, and MATLAB to analyze the measured data. Two different damage cases, namely, uncontrolled naturally damage and controlled damage with additional mass and beam, are used to test the performance of the proposed method.

\section{Case 1: Uncontrolled damage detection}

The experiments are carried out in three stages. First of all, a sensor array including three vibrational transducers is implemented at the root region of every blade. Secondly, hammer tappings are consistently applied to three individual locations at every blade which produces arbitrary excitation inputs. Thirdly, the vibration signals are measured through the DA devices with a sampling rate of $100 \mathrm{kHz}$. The identical procedure is repeated for the other two blades of which the sensor array and the hammer tapping locations remain the same. Fig.6 also shows the experiment setup, the detailed position of the sensor array and the hammer tapping locations. To demonstrate the performance of the proposed WPETF method, the FTF and WETF methods are compared. For the wavelet-based WETF and WPETF methods, db4 (the order 4 Daubechies) is chosen as the wavelet filter function, and a 4-layer decomposition is used. Further, the wavelet energy calculation uses the same setting with the simulation example, namely, 1000 wavelet coefficients as a group.

The results of the FTF, the WETF and the WPETF methods for the three blades are shown in Fig.7. The first row presents the FTF results, showing that the low frequency zone has smooth curves and similar amplitudes. However, the high frequency curves contain significant variations, which may indicate that these parts are very sensitive to noise. Further, the FTF results do not show the visible differences among the healthy Blade \#1 and the damaged Blades \#2 $\&$ \#3. The second row presents the WETF results, clearly showing that both low and high frequency parts behave smoothly. High amplitude occurs at low frequencies, while the amplitude dramatically decreases with frequency increases. This result is expected as the dynamic properties of the blade structure are intrinsically expressed at low frequencies. The blades with different damage levels produce different dynamic properties, namely, different amplitudes in the low and middle frequency zones. When comparing the results from Blade \#1 (healthy condition), Blade \#2 (slightly damaged) and Blade \#3 (severely damaged), it can be seen that the results from Blade \#2 and \#3 have additional spikes enclosed in green rectangles. These additional spikes are the damage indicators since the damage can change the transmissibility functions. These unique spikes also result in lower correlation values compared with the healthy Blade \#1, and enable to distinguish the damaged blades from the healthy one. The third row shows the result of WPETF methods. Clearly this technique adds higher resolution in the middle and high frequency zones. Compared with the WETF method, the WPETF method also provides another spike related to damage in the middle frequency zone, but it has more detailed amplitude variations in the second spike, which enables to distinguish the healthy, less damaged, and severely damaged blades easily. Additionally, compared with FTF, the WETF and WPETF methods have clearly less oscillations in high frequency range, which demonstrates the low sensitivity to noise.

TABLE II Mean correlations with mean variances between the three blades

\begin{tabular}{c|ccc}
\hline Methods & $\begin{array}{c}\text { Blade \#1 } \\
\text { (healthy) }\end{array}$ & $\begin{array}{c}\text { Blade \#2 } \\
\text { (defective) }\end{array}$ & $\begin{array}{c}\text { Blade \#3 } \\
\text { (defective) }\end{array}$ \\
\hline FTF & $0.952 \pm 0.023$ & $0.895 \pm 0.017$ & $0.840 \pm 0.049$ \\
WETF & $0.969 \pm 0.027$ & $0.692 \pm 0.083$ & $0.412 \pm 0.123$ \\
WPETF & $0.983 \pm 0.010$ & $0.714 \pm 0.019$ & $0.554 \pm 0.097$ \\
\hline Results & Reference & Less damaged & Severe damaged \\
\hline
\end{tabular}

To further quantify the blade fault detection results, 6 repeated experiments are conducted. Table II shows the mean correlation with mean variances from six more experiments between the healthy blade (Blade \#1) and the damaged blades (Blade \#2 \& \#3). It is clear that the FTF method has similar correlation values with no significant differences among healthy and damaged blades. These two damaged blades may easily be mis-classified as the healthy ones. However, for WETF and WPETF, the correlations for Blade \#2 \& \#3 are obviously smaller than that of Blade \#1. This indicates that these two blades are in unhealthy conditions. Namely, the wavelet-based methods including both WETF and WPETF perform better than FTF. Furthermore, the correlation value for Blade \#3 is smaller than that of Blade \#2, which means Blade \#3 has severer damage compared with Blade \#2. Apart from that, the new WPETF has smaller mean variances for all three blade results compared with WETF. Therefore, the new method can produce more consistent and robust results and this may benefit from the middle and high frequency resolutions and the low sensitivity to noise.

The real blade damage evidences are collected to validate diagnosis results. Fig. 8 shows the observed damage evidences including cracks and delaminations taken from blade \#2 \& \#3. These real damages match the previous diagnostic results that Blade \#3 has worse defects compared with Blade \#2. This validates that the proposed WPETF method can serve as a wind turbine blade damage indicator.

\section{Case 2: Controlled damage detection}

As one of the common failure modes of an in-service wind turbine blade, ice or sand accretion impairs the blade's structural integrity and further affects the aerodynamic performance negatively. Even slight accumulation issues may change the blade surface roughness and lead to complete turbine shutdown [3]. Case 2 is designed to test the effectiveness of the proposed WPETF method in detecting the accumulated icing/sanding conditions with two scenarios. Scenario A focuses on simulating local accretion conditions, and Scenario B extends the simulation to a large blade surface icing/sanding accumulation conditions. 


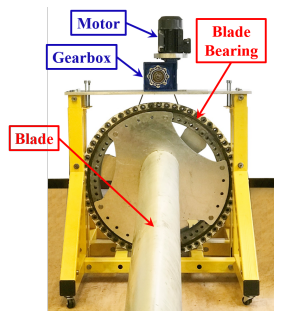

(a) Test-rig front view

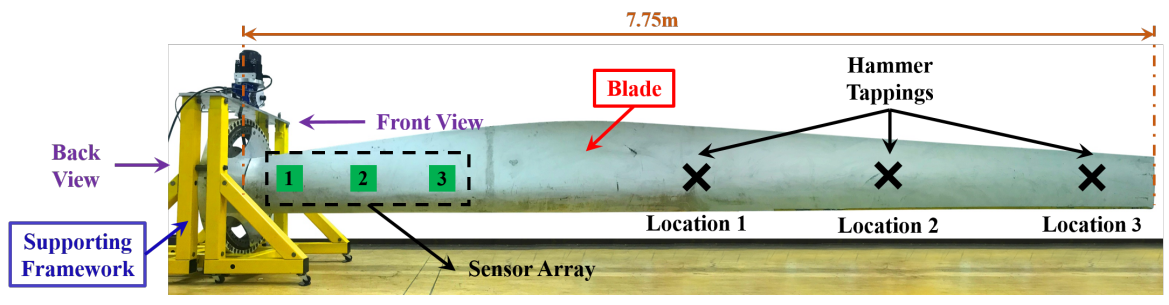

(b) Overall view of the test-rig

Fig. 6 Integrated test-rig setup with front and overall view

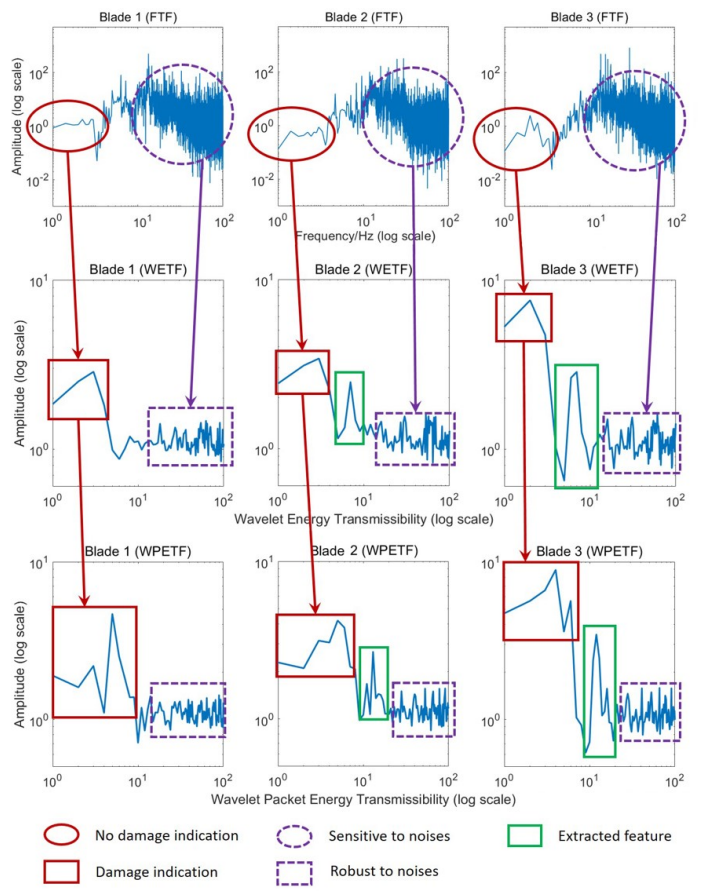

Fig. 7 FTF, WETF and WPETF results of three wind turbine blades

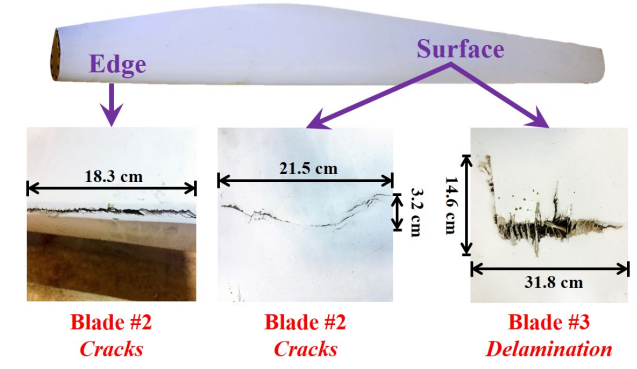

Fig. 8 Damage occurring within test region of WT blade \#2 and \#3

Scenario A: local icing/sanding accretion: Three weights of masses $(2 \mathrm{~kg}, 4 \mathrm{~kg}, 6 \mathrm{~kg})$ are mounted on the surface of the healthy blade between sensors to simulate local accretion of different damage levels. Hammer tapping at the tip of the blade to generate arbitrary excitation signals. Fig.9 shows 6 individual tests result of the WETF method. The results present high repeatability which verifies the reliability of the measured signals. Clearly, additional mass results in unique spikes which indicates the damage. With the adding weight of masses, the
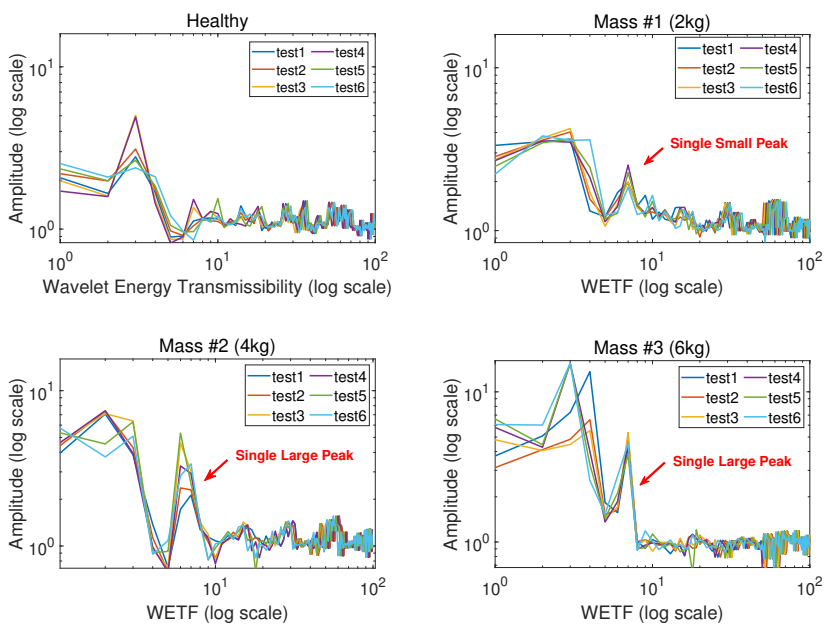

Fig. 9 WETF results of 6 individual tests under additional mass cases

TABLE III Mean correlations with mean variances between the three masses

\begin{tabular}{c|cccc}
\hline Methods & Healthy & $\begin{array}{c}\text { Mass \#1 } \\
(2 \mathrm{~kg})\end{array}$ & $\begin{array}{c}\text { Mass \#2 } \\
(4 \mathrm{~kg})\end{array}$ & $\begin{array}{c}\text { Mass \#3 } \\
(6 \mathrm{~kg})\end{array}$ \\
\hline WETF & $0.935 \pm 0.087$ & $0.747 \pm 0.090$ & $0.502 \pm 0.077$ & $0.395 \pm 0.063$ \\
WPETF & $0.844 \pm 0.014$ & $0.645 \pm 0.020$ & $0.491 \pm 0.059$ & $0.334 \pm 0.048$ \\
\hline Damage Level & Reference & Minor & Medium & Severe \\
\hline
\end{tabular}

amplitude of the spike increase as the mass is increased. Fig.10 displays the results of the WPETF method for the same experiments. By comparison, we can draw a similar conclusion that the mass levels can be revealed explicitly by both the WETF and WPETF methods. However, WPETF provides multiple spikes due to the higher resolution which may provide richer information for icing/sanding detection.

To quantify the identified damage severity, Table III shows the mean correlations with mean variances between the three mass cases. It is clear that both the WPETF and WETF methods can indicate icing/sanding conditions and their relative levels with difference masses. Compared with WETF, WPETF generates consistent results with smaller mean variances values, indicating robust diagnostic results and better noise rejection performance.

Scenario B: large area icing/sanding accretion: As icing or sanding accumulation typically happens alongside the span of the blade and along the leading edge, this scenario uses a beam (length: $1.00 \mathrm{~m}$; width: $0.30 \mathrm{~m}$; thickness: $0.02 \mathrm{~m}$ ) 

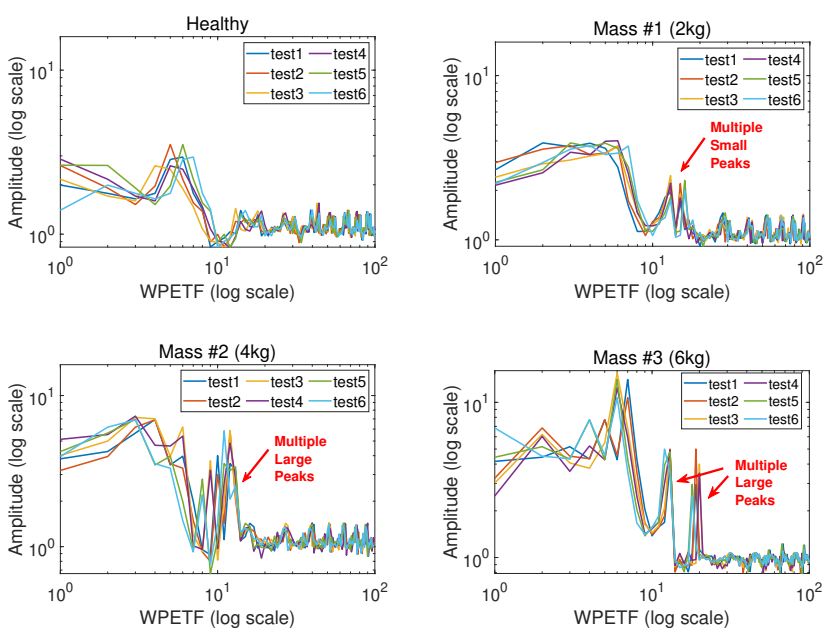

Fig. 10 WPETF results of 6 individual tests under additional mass cases

TABLE IV Mean correlations with mean variances of the covered beam

\begin{tabular}{c|cc}
\hline Methods & Healthy & Beam Region \\
\hline WETF & $0.943 \pm 0.041$ & $0.770 \pm 0.069$ \\
WPETF & $0.881 \pm 0.057$ & $0.505 \pm 0.057$ \\
HHTF & $0.937 \pm 0.094$ & $0.848 \pm 0.053$ \\
HHETF & $0.819 \pm 0.069$ & $0.438 \pm 0.061$ \\
\hline
\end{tabular}

mounted at the blade leading edge to simulate the large area accretion conditions. Hammer tapping also provides arbitrary excitation at the blade tip. This scenario not only compares the new WPETF with WETF but also with another popular time-frequency method, the Hilbert-Huang Transform (HHT). In addition, the energy transmissibility function is extended to HHT transform, producing the new Hilbert-Huang Energy TF (HHETF) method, for additional comparison. Hilbert-Huang transform performs using both Empirical Mode Decomposition (EMD) and Hilbert Transform [29].

Fig. 11 presents 6 individual results of the WETF and WPETF methods for large area damage detection. By comparison, they both present unique spikes which indicates the beam-covered damage. The WPETF result shows multiple spikes rather than a single spike of the WETF method. This also verifies the WPETF's ability to enhance the resolutions. Fig. 12 shows the 6 individual damage detection results for HHT and HHETF methods. Clearly, HHT is not able to detect the blade damage. With the integration of energy transmissibility function with HHT, the new HHETF method presents the differences between healthy and damaged conditions and therefore it can detect the blade faults. However, it has multiple and overlapped spikes and has less robust results compared with that of WPETF. Table IV provides the mean correlations with mean variances of the beam-covered tests. Scenario B has the same conclusion with Scenario A where the proposed WPETF produces more consistent and robust results than other three methods.
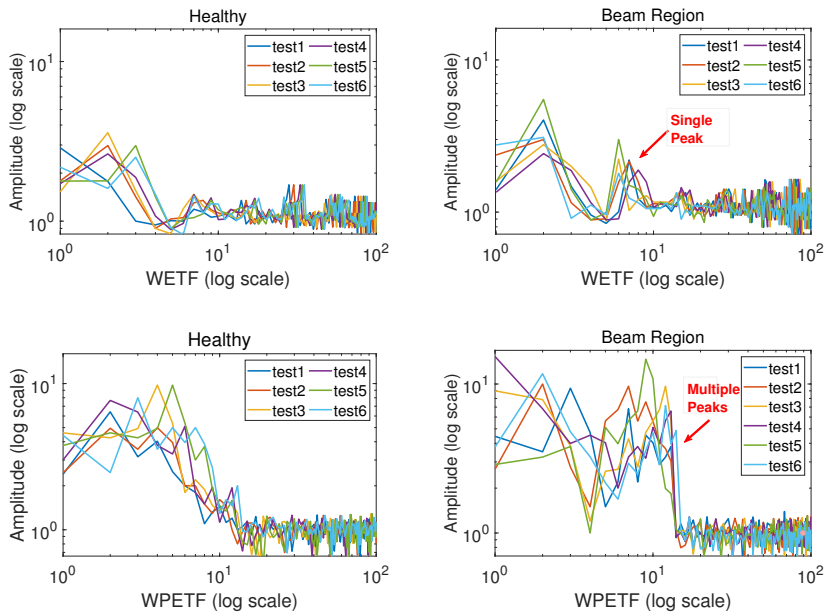

Fig. 11 WETF and WPETF results of 6 individual tests of large area icing accretion detection
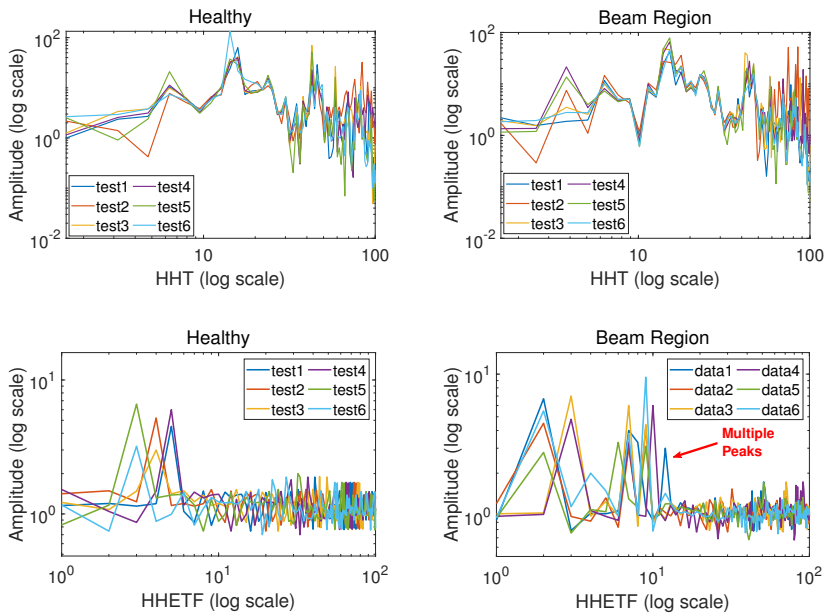

Fig. 12 HHTF and HHETF results of 6 individual tests of large area icing accretion detection

\section{DISCUSSION}

Some discussions related to the use of the proposed method and its wide applications are listed as follows.

- Choose of wavelet basis functions: Compared with the $\mathrm{db} 4$ wavelet basis function used in this paper, other functions such as Haar and Mexican Hat essentially are band-pass filters. Hence, they can also produce similar results with slight differences in transmissibility functions.

- Computational complexity: RMS is applied to different segments of data when calculating WPETF, which leads to less numerical operation compared with conventional FTF methods. Thus, the WPETF performs faster than FTF and demands less computational resources. It can be implemented off or online for operational in-situ wind turbine blades. For online condition monitoring, wireless transmission techniques are preferred for sensing data collection to avoid cable connection.

- Other applications: Although this paper considers the 
application in wind turbine blade fault detection, the proposed method can be used and easily transferred to other similar condition assessment scenarios such as airfoil, rails or bridges.

- Future work: In this paper, the WPETF method is proposed to detect the blade damage and identify damage severity levels. It has the potential to identify different failure modes of the blade due to the fact that various blade fault types produce different amplitudes in certain frequency ranges of the energy TF functions. Future work will investigate the diagnosis of failure modes, qualification of damage levels and estimation of the blade remaining lifespan with the proposed framework.

\section{CONCLUSION}

In this paper, a novel Wavelet Package Energy Transmissibility Function (WPETF) method is proposed for wind turbine blade damage detection. A mass-damping-spring system is introduced as a numerical example to illustrate that compared with conventional Transmissibility Function (TF) methods including Fourier TF (FTF) and Wavelet Energy TF (WETF) methods, the WPETF method adds more resolution to high frequency range and maintain low sensitivity to noise. Further, uncontrolled damage experiments use three naturally-damaged industrial-scale wind turbine blades to demonstrate the performance of the WPETF methods for damage detection. Controlled damage experiments using additional mass simulate the practical blade surface ice/sand accretions. In both cases, the proposed WPETF method effectively detects the blade faults and clearly indicate damage levels. Compared with the WETF method, WPETF provides richer information in interested frequency zones and produces more consistent and robust diagnosis results.

\section{APPENDIX}

Analysis of low sensitivity to noise: Assume $\mathrm{x}=$ $\left[x_{1}, \ldots, x_{i}, \ldots, x_{m}\right]$ and $\mathbf{y}=\left[y_{1}, \ldots y_{i}, \ldots, y_{m}\right]$, are WD (or WPD) of two segments of wavelet coefficients from two different measured responses, where $z, z=1, \ldots, Z$, and $m$ is the data length. Firstly, consider noise-free wavelet energy $\mathrm{TF}$ of the two responses for this specific segment:

$$
T=\frac{R M S(\mathbf{x})}{R M S(\mathbf{y})}=\frac{\|\mathbf{x}\|_{2}}{\|\mathbf{y}\|_{2}}
$$

Then consider the noisy wavelet energy TF:

$$
\tilde{T}=\frac{\|\mathbf{x}+\boldsymbol{\Theta}\|_{2}}{\left\|\mathbf{y}+\boldsymbol{\Theta}^{\prime}\right\|_{2}}
$$

where $\boldsymbol{\Theta}=\left[\theta_{1}, \ldots, \theta_{i}, \ldots, \theta_{m}\right]$ is a Gaussian white noise vector with zero mean and variance $\sigma^{2}=\frac{1}{m} \sum_{i=1}^{m} \theta_{i}^{2}$, and $\Theta^{\prime}=\left[\theta^{\prime}{ }_{1}, \ldots, \theta^{\prime}{ }_{i}, \ldots, \theta^{\prime}{ }_{m}\right]$ is also a Gaussian white noise vector with zero mean and variance ${\sigma^{\prime}}^{2}=\frac{1}{m} \sum_{i=1}^{m} \theta^{\prime}{ }_{i}{ }^{2}$, namely, $\|\boldsymbol{\Theta}\|_{2}=m \sigma^{2}$ and $\left\|\Theta^{\prime}\right\|_{2}=m{\sigma^{\prime}}^{2}$. Let $\Delta T$ denote the difference between noise-free wavelet energy TF $T$ and noisy wavelet energy TF $\tilde{T}$, then

$$
|\Delta T|=|\tilde{T}-T|=\frac{\tilde{T}^{2}-T^{2}}{|\tilde{T}+T|}
$$

$$
\begin{aligned}
|\Delta T| & =\frac{\left|\sum_{i=1}^{m} \sum_{j=1}^{m}\left(x_{i}+\theta_{i}\right)^{2} y_{j}^{2}-\sum_{i=1}^{m} \sum_{j=1}^{m}\left(y_{j}+\theta^{\prime}{ }_{j}\right)^{2} x_{i}^{2}\right|}{|\tilde{T}+T|\left\|\mathbf{y}+\mathbf{\Theta}^{\prime}\right\|_{2}\|\mathbf{y}\|_{2}} \\
& =\frac{\left|\sum_{i=1}^{m} \sum_{j=1}^{m}\left(2 x_{i} y_{j}+\theta_{i} y_{j}+\theta^{\prime}{ }_{j} x_{i}\right)\left(\theta_{i} y_{j}-\theta^{\prime}{ }_{j} x_{i}\right)\right|}{|\tilde{T}+T|\left\|\mathbf{y}+\boldsymbol{\Theta}^{\prime}\right\|_{2}\|\mathbf{y}\|_{2}}
\end{aligned}
$$

For simplicity, denote the denominator of equation (24) as $\Omega=|\tilde{T}+T|\left\|\mathbf{y}+\boldsymbol{\Theta}^{\prime}\right\|_{2}\|\mathbf{y}\|_{2}$. Since $\mathbf{x}$ and $\mathbf{y}$ are all bounded, let $X \geq x_{i}^{2}$, and $Y \geq y_{j}^{2}$, where $i, j=1, \ldots, m, i \neq j$ and $X>0, Y>0$. Since $\Theta$ and $\Theta^{\prime}$ are bounded, let $X_{\Theta} \geq$ $\left(x_{i}+\theta_{i}\right)^{2}$ and $Y_{\Theta^{\prime}} \geq\left(y_{j}+\theta^{\prime}{ }_{j}\right)^{2}, X_{\Theta}>0, Y_{\Theta^{\prime}}>0$. Denote the minimal value of $\Omega$ as $\Pi$, then

$$
\begin{gathered}
\left\|\mathbf{y}+\boldsymbol{\Theta}^{\prime}\right\|_{2} \leq \sqrt{m Y_{\Theta^{\prime}}} \\
\|\mathbf{y}\|_{2} \leq \sqrt{m Y} \\
\Omega=|\tilde{T}+T|\left\|\mathbf{y}+\boldsymbol{\Theta}^{\prime}\right\|_{2}\|\mathbf{y}\|_{2} \\
\geq\left(\sqrt{\frac{X_{\Theta}}{Y_{\Theta^{\prime}}}}+\sqrt{\frac{X}{Y}}\right) Y_{\Theta^{\prime}} Y=\Pi
\end{gathered}
$$

Let $\Xi=\left|2 x_{i} y_{j}+\theta_{i} y_{j}+\theta^{\prime}{ }_{j} x_{i}\right|$, where $i, j=1, \ldots, m$ and denote the maximal absolute value of $\Xi$ is $\Gamma$. Since $\Theta, \Theta^{\prime}, \mathbf{x}$ and $\mathbf{y}$ are all bounded, their maximum values are denoted as $\theta^{*}, \theta^{\prime *}, X^{*}$ and $Y^{*}$ respectively. Then,

$$
\Xi \leq 2 X^{*} Y^{*}+\theta^{*} Y^{*}+\theta^{\prime *} X^{*}=\Gamma
$$

Equation (24) is then bounded by

$$
|\Delta T| \leq \frac{\Gamma}{\Pi}\left|\boldsymbol{\Theta} \cdot \mathbf{y}-\boldsymbol{\Theta}^{\prime} \cdot \mathbf{x}\right| \leq \frac{\Gamma}{\Pi}\left(|\boldsymbol{\Theta}| Y^{*}+\left|\Theta^{\prime}\right| X^{*}\right)
$$

In other words, the difference between noise-free and noisy case is bounded. Some discussions related to the noise rejection properties are given below:

- Although the above analysis of noise rejection is suitable for the noise in both low and high frequency ranges, the high frequency noise is often more significant as most of the engineering applications have large signals in the low frequency parts, but have smaller signals in the high frequency parts.

- For the wavelet energy TF method, as it uses the RMS value of a group of wavelet coefficients, the associated noise is also grouped and the final noise influence is related to the variance of the grouped noise instead of each single noise. However, for the FTF method, it uses single frequency component when estimating the TFs, and its results vary with each single noise. Therefore, the FTF method has more oscillating results. This is also demonstrated in both simulated and experimental results.

\section{REFERENCES}

[1] M. O. Hansen, "Aerodynamics of wind turbines: rotors, loads and structure," vol. 17, pp. 95-97, 2000

[2] M. Klitgaard, C. F. Andersen, M. Ravn, C. Skovby, S. B. Bojesen, and B. Peter, "Wind turbine blade and an associated manufacturing method," Jun. 11 2019, US Patent 10,316,817. 
[3] Z. Liu and L. Zhang, "A review of failure modes, condition monitoring and fault diagnosis methods for large-scale wind turbine bearings," Measurement, vol. 149, p. 107002, 2019.

[4] W. Yang, Z. Lang, and W. Tian, "Condition monitoring and damage location of wind turbine blades by frequency response transmissibility analysis," IEEE Transactions on Industrial Electronics, vol. 62, no. 10, pp. 6558-6564, 2015.

[5] J. Ribrant and L. Bertling, "Survey of failures in wind power systems with focus on Swedish wind power plants during 1997-2005," in 2007 IEEE Power Engineering Society General Meeting. IEEE, 2007, pp $1-8$.

[6] J. Wei and J. McCarty, "Acoustic emission evaluation of composite wind turbine blades during fatigue testing," Wind Engineering, pp. 266-274, 1993.

[7] W. Yang, "Testing and condition monitoring of composite wind turbine blades," Recent Advances in Composite Materials for Wind Turbine Blade, World Academic Publishing, Hong Kong, pp. 147-169, 2013.

[8] W. Yang, P. J. Tavner, C. J. Crabtree, Y. Feng, and Y. Qiu, "Wind turbine condition monitoring: technical and commercial challenges,' Wind Energy, vol. 17, no. 5, pp. 673-693, 2014.

[9] M. Rumsey and W. Musial, "Application of infrared thermography nondestructive testing during wind turbine blade tests," Transactions-American Society of Mechanical Engineers Journal of Solar Energy Engineering, vol. 123, no. 4, pp. 271-271, 2001.

[10] G. Ye, B. Neal, A. Boot, V. Kappatos, C. Selcuk, and T.-H. Gan, "Development of an Ultrasonic NDT System for Automated In-Situ Inspection of Wind Turbine Blades." IFFSTTAR, Inria, Université de Nantes, Jul. 2014

[11] F. Grasse, V. Trappe, S. Thöns, and S. Said, "Structural health monitoring of wind turbine blades by strain measurement and vibration analysis," 8th International conference on structural dynamics, vol. MS25 Tue, pp. 3490-3497, 2011.

[12] B. Lu, Y. Li, X. Wu, and Z. Yang, "A review of recent advances in wind turbine condition monitoring and fault diagnosis," in 2009 IEEE Power Electronics and Machines in Wind Applications. IEEE, 2009, pp. 1-7.

[13] Z. Hameed, Y. Hong, Y. Cho, S. Ahn, and C. Song, "Condition monitoring and fault detection of wind turbines and related algorithms: A review," Renewable and Sustainable energy reviews, vol. 13, no. 1, pp. 1-39, 2009.

[14] Y. Li, X. Wang, Z. Liu, X. Liang, and S. Si, "The entropy algorithm and its variants in the fault diagnosis of rotating machinery: A review,' Ieee Access, vol. 6, pp. 66723-66741, 2018.

[15] T. D. Popescu and D. Aiordachioaie, "Fault detection of rolling element bearings using optimal segmentation of vibrating signals," Mechanical Systems and Signal Processing, vol. 116, pp. 370-391, 2019.

[16] H. Wang and X. Jing, "Vibration signal-based fault diagnosis in complex structures: A beam-like structure approach," Structural Health Monitoring, vol. 17, no. 3, pp. 472-493, 2018.

[17] J. P. Amezquita-Sanchez and H. Adeli, "Signal processing techniques for vibration-based health monitoring of smart structures," Archives of Computational Methods in Engineering, vol. 23, no. 1, pp. 1-15, 2016.

[18] N. Laouti, N. Sheibat-Othman, and S. Othman, "Support vector machines for fault detection in wind turbines," IFAC Proceedings Volumes, vol. 44, no. 1, pp. 7067-7072, 2011.

[19] Y. Du, S. Zhou, X. Jing, Y. Peng, H. Wu, and N. Kwok, "Damage detection techniques for wind turbine blades: A review," Mechanical Systems and Signal Processing, vol. 141, p. 106445, 2020.

[20] G. J. Herbert, S. Iniyan, E. Sreevalsan, and S. Rajapandian, "A review of wind energy technologies," Renewable and sustainable energy Reviews, vol. 11, no. 6, pp. 1117-1145, 2007.

[21] D. Auger, Q. Wang, J. Trevelyan, S. Huang, and W. Zhao, "Investigating the quality inspection process of offshore wind turbine blades using b-spline surfaces," Measurement, vol. 115, pp. 162-172, 2018.

[22] H. Zoubek, S. Villwock, and M. Pacas, "Frequency response analysis for rolling-bearing damage diagnosis," IEEE Transactions on Industrial Electronics, vol. 55, no. 12, pp. 4270-4276, 2008.

[23] N. M. Maia, R. A. Almeida, A. P. Urgueira, and R. P. Sampaio, "Damage detection and quantification using transmissibility," Mechanical Systems and Signal Processing, vol. 25, no. 7, pp. 2475-2483, 2011.

[24] L. Zhang and Z. Q. Lang, "Wavelet energy transmissibility function and its application to wind turbine bearing condition monitoring," IEEE Transactions on Sustainable Energy, vol. 9, no. 4, pp. 1833-1843, 2018.

[25] D. E. Newland, "Wavelet analysis of vibration: part 1-theory," Journal of vibration and acoustics, vol. 116, no. 4, pp. 409-416, 1994.

[26] N. M. Maia, R. A. Almeida, A. P. Urgueira, and R. P. Sampaio, "Damage detection and quantification using transmissibility," Mechanical Systems and Signal Processing, vol. 25, no. 7, pp. 2475-2483, 2011.
[27] H. Zhengjia, Z. Jiyuan, H. Yibin, and M. Qingfeng, "Wavelet transform and multiresolution signal decomposition for machinery monitoring and diagnosis," in Proceedings of the IEEE International Conference on Industrial Technology (ICIT'96). IEEE, 1996, pp. 724-727.

[28] Y. Lage, M. Neves, N. Maia, and D. Tcherniak, "Force transmissibility versus displacement transmissibility," Journal of Sound and Vibration, vol. 333, no. 22, pp. 5708-5722, 2014

[29] N. E. Huang and Z. Wu, "A review on hilbert-huang transform: Method and its applications to geophysical studies," Reviews of geophysics, vol. 46, no. 2, 2008 . 